\title{
Accessory Respiratory Muscle Activation during Chest Expansion Exercise using Elastic Bands in Children with Cerebral Palsy
}

\author{
Seung-Oh Shin, PT • Nan-Soo Kim PT, PhD ${ }^{1+}$
}

Department of Physical Therapy, Graduate School, Catholic University of Pusan

${ }^{1}$ Department of Physical Therapy, College of Health Sciences, Catholic University of Pusan

Received: July 27, 2016 / Revised: July 31, 2016 / Accepted: August 8, 2016

(C) 2016 J Korean Soc Phys Med

\section{| Abstract |}

PURPOSE: The aim of this study was to evaluate activation of accessory respiratory muscles using electromyography during chest expansion upper extremity flexion, abduction, and external rotation exercises with an elastic band in children with cerebral palsy.

METHODS: The subjects were 10 children with cerebral palsy. The inclusion criterion for participation was a Gross Motor Function Classification System level of I to III. The subjects were instructed to perform upper extremity flexion, abduction, and external rotation exercises with inspiration, and extension, adduction, and internal rotation exercises with expiration while seated on a chair without a backrest. PM (Pectoralis major), SCM (sternocleidomastoid), RA (rectus abdominis), and EO (external oblique) muscle activities were measured using electromyography.

RESULTS: All tested muscles showed a statistically significant increase in activity after elastic band exercise. There were significant differences in PM, SCM, RA, and EO

†Corresponding Author : hnskim@cup.ac.kr

This is an Open Access article distributed under the terms of the Creative Commons Attribution Non-Commercial License (http://creativecommons.org/licenses/by-nc/3.0) which permits unrestricted non-commercial use, distribution, and reproduction in any medium, provided the original work is properly cited. results after chest expansion exercise using elastic band. SCM showed the largest increase in activity after use of elastic bands, at $52.37 \pm 45.88 \%$, followed by the RA (50.56 \pm 79.31 ), EO (35.42 \pm 35.45$)$, and PM (31.72 \pm 25.64$)$. The increase in the SCM was greatest, followed by increases in the RA, EO, and PM

CONCLUSION: These finding suggest that activity of accessory respiratory muscles increases with use of elastic bands during chest expansion exercise in cerebral palsy.

Key Words: Accessory Respiratory muscle, Cerebral palsy, Chest expansion, Elastic band

\section{Introduction}

Cerebral palsy is a major disease in children, and causes posture and activity disorders. It is associated with brain injury in utero or during birth (Bax et al., 2005). Cerebral palsy in children involved musculoskeletal deformities, shortening and dystrophic changes in muscles, weakness of the entire body, respiratory muscle weakness, asymmetric thoracic growth, and impaired respiratory function, resulting in increased respiratory infections and mortality (Toder, 2000). 
Respiratory muscle weakness causes breathing difficulty when performing activity. Therefore, intervention is needed to strengthen respiratory muscles, reduce dyspnea, and enhance exercise tolerance (Mulreany et al., 2003). Physical therapy used to strengthen the respiratory muscles and enhance pulmonary function includes chest expansion resistance training, abdominal breathing, and pursed-lip breathing (Kisner and Collby, 2012). Individuals who experience difficulty breathing tend to compensate by excessive use of accessory respiratory muscles, including the PM and SCM during inspiration, and RA, EO, and internal oblique (IO) during expiration (Moreno et al., 2005; Kendall et al., 2005; Ratnovsky et al., 2008).

With upper extremity flexion, abduction, and external rotation against resistance, the major respiratory muscles (the diaphragm and intercostal muscles) are activated (Moreno et al., 2005) together with the accessory respiratory muscles the PM and serratus anterior (Sullivan and Portney, 1980), which helps expand the chest and improve breathing (Areas et al., 2013).

Since resistance training enhances muscle strength and endurance, improves oxygen carrying capacity, modifies muscle type, and decreases muscle fatigue in orthopedic patients, it is also often used in patients with cardiovascular disease (Bennell and Hinman, 2011; Braith and Beck, 2008). Children with cerebral palsy receive direct manual resistance therapy and training with a barbell or other equipment. However, the degree of resistance applied by therapists is variable, and difficulty with objective measurement can arise when using such methods. Resistance training using elastic bands has recently been widely used to compensate for these limitations (Ham, 2000; Choi et al., 2010; Lin and Sung, 2012; Areas et al., 2013). Elastic bands are convenient, economical, and safe to use, and provide resistance without relying on gravity; elastic bands do not impose spatial limitations, and are commonly used in clinical practice (Simoneau et al., 2001).
Electromyography (EMG) during chest expansion with accessory respiratory muscles is commonly used in patients with respiratory diseases (Ng and Stokes, 1992; Maarsingh et al., 2000).

This study therefore aims to evaluate upper extremity flexion, abduction, and external rotation against resistance in children with cerebral palsy, and to investigate changes in activation of accessory respiratory muscles during chest expansion.

\section{Methods}

\section{Subjects}

This study was conducted in 10 children with cerebral palsy who agreed to participate in the research (boys: 5 , girls: 5)

The inclusion criterion for participation was a Gross Motor Function Classification System level of I to III. The average age, height, and weight of participants was $8.10 \pm 3.25$ years old, $114.18 \pm 13.37 \mathrm{~cm}$, and $23.14 \pm 8.47 \mathrm{~kg}$.

\section{Procedure}

All subjects performed chest expansion exercises with upper extremity flexion, abduction, and external rotation while knee does not widen on seated on a chair without an armrest and backrest. The subjects were instructed to: 1) breathe in and out at rest; then, 2) perform upper extremity flexion, abduction, and external rotation with inspiration, and extension, adduction, and internal rotation with expiration without a yellow Thera-Band (Hygenic Corporation, Akron, OH, USA). and then, 3) perform the same exercises with a yellow Thera-Band.

For data collection, subjects performed three trials of each exercise with a 1-min rest period between trials. The EMG signals collected during the three exercises were expressed as a percentage of the calculated root mean square of a reference voluntary contraction $(\% \mathrm{RVC})$. 


\section{Data collection and EMG processing}

Surface EMG (LXM5308, LAXTHA Inc., Korea) was used to record activity of the PM, SCM, RA, and EO muscles. Disposable bipolar $\mathrm{Ag} / \mathrm{AgCl}$ surface electrodes were positioned at an inter-electrode distance of $2 \mathrm{~cm}$. Prior to applying the electrodes, skin impedance was reduced by cleansing with an alcohol swab. Electrode placements for each muscle were as follows: PM - (sternal fibers) located on the chest wall parallel to the muscle origin (approximately $2 \mathrm{~cm}$ lateral to the axillary fold); SCM placed at lower $1 / 3$ of the line connecting sternal notch and mastoid process; RA - $3 \mathrm{~cm}$ superior to the umbilicus and $2 \mathrm{~cm}$ lateral to the midline; $\mathrm{EO}$ - an oblique arrangement above the anterior superior iliac spine and lateral to the umbilicus (Cram et al., 1998; Falla et al., 2002).

The EMG signals were band-pass filtered from 20 to $500 \mathrm{~Hz}$ and sampled at $1024 \mathrm{~Hz}$. Following data collection, EMG signals were rectified and smoothed using the root mean square (RMS) for raw data. RVCs were collected to normalize the EMG data of the PM, SCM, RA, and EO.

\section{Data analysis}

Collected data were analyzed using SPSS version 22.0 (SPSS, Inc., Chicago, IL, USA). All data showed non-normal distributions according to the KolmogorovSmirnov test. Wilcoxon signed-rank tests were used to compare differences in muscle activity between two conditions (Non-band vs. Band exercise)

\section{Results}

\section{EMG activities}

EMG activities of each muscle tested during the chest expansion exercises with upper extremity flexion, abduction, and external rotation are summarized in Table 1.

There were significant differences in PM, SCM, RA, and EO results after chest expansion exercise using elastic band. SCM showed the largest increase in activity after use of elastic bands, at $52.37 \pm 45.88 \%$, followed by the RA (50.56 \pm 79.31$)$, EO (35.42 \pm 35.45$)$, and PM (31.72 \pm 25.64).

\section{Discussion}

Children with cerebral palsy have impaired breathing. The diaphragm contracts excessively during inspiration to compensate for weakened respiratory muscles, the lower rib cage flares outward, and the sternum is retracted because it cannot overcome the strong pulling force. This causes the mid-thorax to retract, giving the chest a funnel-like appearance (Crystal, 1997). Due to impaired breathing, patients tend to compensate by excessive use of the accessory respiratory muscles, including the PM and SCM during inspiration, and RA, EO, and IO during expiration (Moreno et al., 2005; Kendall et al., 2005; Ratnovsky et al., 2008). With upper extremity flexion, abduction, and

Table 1. Electromyography activities (\%RVC) during chest expansion with upper extremity flexion, abduction, and external rotation

(unit: \%RVC)

\begin{tabular}{llll}
\hline & Non-band & Band & P value \\
\hline PM & $50.89 \pm 47.76$ & $104.94 \pm 82.42$ & $.01^{* *}$ \\
\hline SCM & $167.12 \pm 130.53$ & $295.45 \pm 179.23$ & $.01^{* *}$ \\
\hline RA & $13.16 \pm 18.75$ & $65.47 \pm 75.25$ & $.01^{* *}$ \\
\hline EO & $16.35 \pm 13.02$ & $65.51 \pm 49.11$ & $.02^{*}$ \\
\hline
\end{tabular}

Mean \pm SD

PM: pectoralis major, SCM: sternocleidomastoid, RA: rectus abdominis, EO: external oblique 
external rotation against resistance, the major breathing muscles (diaphragm and intercostal muscles) are activated (Moreno et al., 2005) together with the accessory respiratory muscles (PM and serratus anterior) which expands the chest and improves breathing (Areas et al., 2013; Sullivan and Portney, 1980). In addition Sullivan and Portney (1980) studied the electromyographic activity of various upper limb PNF patterns (upper extremity flexion, abduction, and external rotation) and described that the same movement used in this study activates the muscles of the pectoralis major and serratus anterior, both accessory respiratory muscles, and may possibly have an effect on the increase in respiratory muscle activity. However, there has been a lack of research on muscle activation in the accessory respiratory muscles in children with cerebral palsy.

Therefore, this study implemented a chest expansion exercise in children with cerebral palsy, in order to investigate whether resistance training using elastic bands affects muscle activation in accessory respiratory muscles.

The study showed that muscle activity of SCM was greatest when elastic bands were not used in chest expansion exercise, and this was statistically significant. This result indicates that during the study, the SCM was used as an accessory muscle more than the PM, with upper extremity flexion, abduction, and external rotation during inspiration. As Czaplinski et al. (2006) reported, the SCM acts as an upper torso and accessory inspiratory muscle that raises the thorax during inspiration, expands lung volume, and therefore affects forced vital capacity. Areas et al. (2013) reported a positive effect on respiratory muscle strength with upper extremity flexion, abduction, and external rotation exercise using elastic bands.

Trunk stability is essential for raising and lowering the upper limb while sitting. (Hodges and Richardson, 1997a; Hodges and Richardson, 1997b). Lee et al. (2016) investigated whether elastic band use during upper extremity lifting can activate the ipsilateral external oblique muscle. Jeon (2013) stated that trunk muscle strengthening exercise and use of a trunk belt leads to improved trunk control ability and pulmonary function. Considering that strength is a condition for upper and lower limb movement once trunk stability is achieved, this result shows that the patient recruits as much RA muscle strength as possible to overcome resistance from the elastic band and raise the upper limbs. Choi et al. (2013) also reported that RA muscle strength can alter upper extremity function. Thus, upper limb chest expansion exercises using elastic bands greatly different activation of accessory respiratory muscles in children with cerebral palsy.

This study was limited by the small sample of children with cerebral palsy. Additional studies are needed to investigate the effects on pulmonary function in children with cerebral palsy by measuring respiratory muscle and pulmonary function, in addition to changes in muscle activity.

\section{Conclusion}

In clinical practice, upper extremity flexion, abduction, and external rotation, even without elastic bands, may assist respiration in children with cerebral palsy who have respiratory muscle weakness, by activating accessory respiratory muscles. As the study shows, upper extremity exercise for chest expansion increases PM, SCM, RA, and EO accessory respiratory muscle activities during inspiration and expiration. This suggests that chest expansion exercise using elastic bands can have a positive effect on accessory respiratory muscle activity.

\section{Acknowledgements}

This study was supported by a 2016 research grant from Catholic University of Pusan. 


\section{References}

Areas G, Borghi-Silva A, Lobato AN, et al. Effect of upper extremity proprioceptive neuromuscular facilitation combined with elastic resistance bands on respiratory muscle strength: A randomized controlled trial. Brazilian journal of physical therapy. 2013;17(6): 541-6.

Bax M, Goldstein M, Rosenbaum P, et al. Proposed definition and classification of cerebral palsy, april 2005. Dev Med Child Neurol. 2005;47(8):571-6.

Bennell KL, Hinman RS. A review of the clinical evidence for exercise in osteoarthritis of the hip and knee. J Sci Med Sport. 2011;14(1):4-9.

Braith RW, Beck DT. Resistance exercise: Training adaptations and developing a safe exercise prescription. Heart Fail Rev. 2008;13(1):69-79.

Choi WJ, Kim YK, Son KH. The effects of elastic band combined with proprioceptive neuromuscular facilitation upper extremity patterns on body alignment and weight support in patients with hemiplegia. J Korean soc phys med. 2010;5(1):113-23.

Choi YC, Park SJ, Lee MH, et al. The effects of trunk muscle strengthening exercises on balance performance of sitting posture and upper extremity function of children with spastic diplegic cerebral palsy. J Korean soc phys med. 2013;8(1):117-25.

Cram JR, Kasman GS, Holtz J. Introduction to surface electromyography: Aspen Publishers. 1998.

Crystal RG. The lung: Scientific foundations. Lippincott-Raven. 1997.

Czaplinski A, Yen AA, Appel SH. Forced vital capacity (fvc) as an indicator of survival and disease progression in an als clinic population. J Neurol Neurosurg Psychiatry. 2006;77(3):390-2.

Falla D, Dall'Alba P, Rainoldi A, et al. Location of innervation zones of sternocleidomastoid and scalene muscles a basis for clinical and research electromyography applications. Clin Neurophysiol. 2002;113(1):57-63.

Ham YW. Effects of theraband exercise on isotonic strength of flexor muscles in upper extremities. Journal of health science and medical technology. 2000;26(1): 49-56.

Hodges PW, Richardson CA. Contraction of the abdominal muscles associated with movement of the lower limb. Phys Ther. 1997a;77(2):132-42.

Hodges PW, Richardson CA. Feedforward contraction of transversus abdominis is not influenced by the direction of arm movement. Exp Brain Res. 1997b; 114(2):362-70.

Jeon JY. Effects of the trunk muscle strengthening exercise and using the trunk belt on pulmonary function and trunk control ability for children with spastic cerebral palsy. Master's Degree, Daejeon university. 2013.

Kendall RW, Jacquemin G, Frost R, et al. Creatine supplementation for weak muscles in persons with chronic tetraplegia: A randomized double-blind placebo-controlled crossover trial. J Spinal Cord Med. 2005;28(3):208-13.

Kisner C, Colby LA. Therapeutic exercise: Foundations and techniques. F.A. Davis. 2012.

Lee HO, Bae WS, Shin JW. A comparison of the trunk muscle activity according to the direction of upper extremity lifting using elastic band. J Korean soc phys med. 2016;11(2):25-31.

Lin SF, Sung HC. The effectiveness of resistance training with thera band on physiological functions for older adults: A systematic review. 2012;10(56):10.

Maarsingh EJ, van Eykern LA, Sprikkelman $A B$, et al. Respiratory muscle activity measured with a noninvasive emg technique: Technical aspects and reproducibility. J Appl Physiol. 2000;88(6):1955-61.

Moreno MA, Silva Ed, Goncalves M. The effect of proprioceptive neuromuscular facilitation techniques - kabat method on maximum respiratory pressure. Fisioter Mov. 2005;18(2):53-61. 
Mulreany LT, Weiner DJ, McDonough JM, et al. Noninvasive measurement of the tension-time index in children with neuromuscular disease. J Appl Physiol. 2003;95(3):931-7.

Ng GY, Stokes MJ. Emg recordings of the respiratory muscles during unilateral and bilateral chest expansion. Australian Journal of Physiotherapy. 1992;38(3): 203-8.

Ratnovsky A, Elad D, Halpern P. Mechanics of respiratory muscles. Respir Physiol Neurobiol. 2008;163(1-3): $82-9$.
Simoneau GG, Bereda SM, Sobush DC, et al. Biomechanics of elastic resistance in therapeutic exercise programs. J Orthop Sports Phys Ther. 2001;31(1):16-24.

Sullivan PE, Portney LG. Electromyographic activity of shoulder muscles during unilateral upper extremity proprioceptive neuromuscular facilitation patterns. Phys Ther. 1980;60(3):283-8.

Toder DS. Respiratory problems in the adolescent with developmental delay. Adolesc Med. 2000;11(3): 617-31. 